\title{
Corrigendum
}

\section{Jun dimerization protein 2 is a critical component of the Nrf2/MafK complex regulating the response to ROS homeostasis}

S Tanigawa, CH Lee, CS Lin, CC Ku, H Hasegawa, S Qin, A Kawahara, Y Korenori, K Miyamori, M Noguchi, LH Lee, YC Lin, CL Steve Lin, Y Nakamura, C Jin, N Yamaguchi, R Eckner, D-X Hou and KK Yokoyama

Cell Death and Disease (2014) 5, e1344; doi:10.1038/cddis.2014.322; published online 17 July 2014

Correction to: Cell Death and Disease (2013) 4, e921; doi:10.1038/cddis.2013.448; published online 14 November 2013

Since the publication of this paper, the authors have noticed that there was an error in Figure $2 \mathrm{a}$. The western blotting image was incorrect. The error has now been rectified. The corrected article appears online together with this corrigendum. The authors would like to apologize for any inconvenience this may have caused. 
a

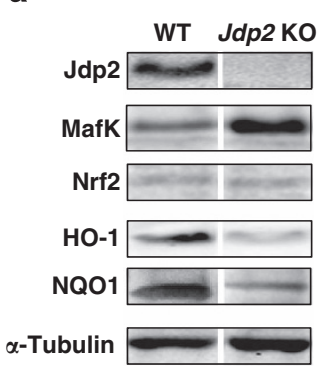

b

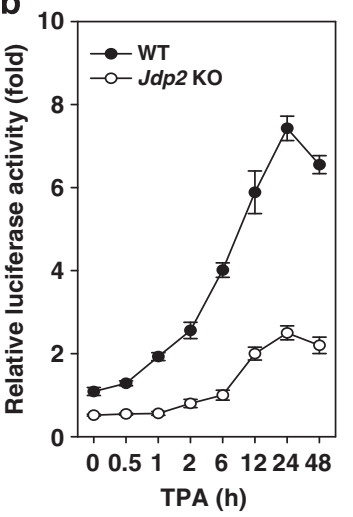

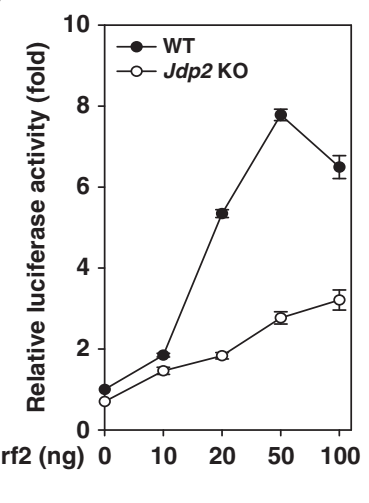

d

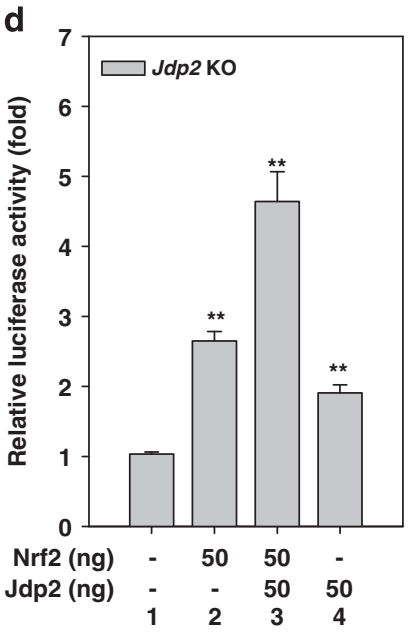

e

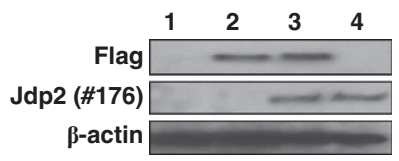

f

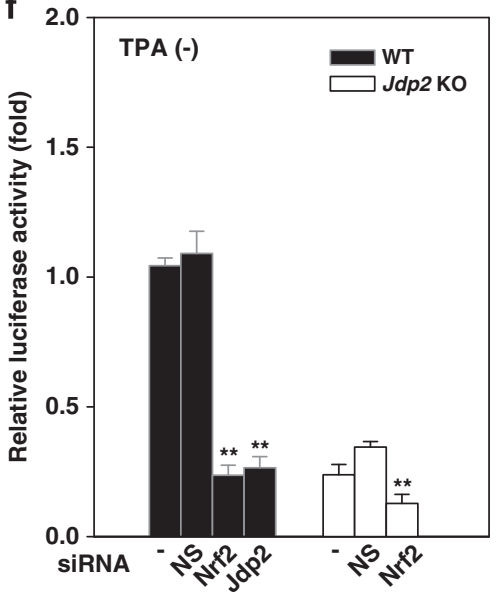

g

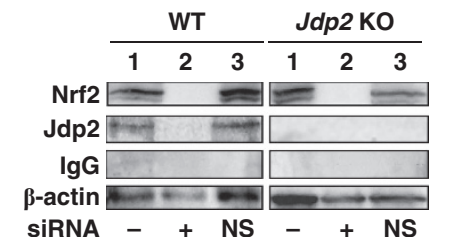

Figure 2 JDP2 is required for ARE activation. (a) Protein detection in WT and Jdp2 KO MEFs using western blotting. The cellular lysates from WT and Jdp2 KO MEFs $(40 \mu \mathrm{g})$ were separated on SDS-PAGE and then transferred onto a membrane. Jdp2, MafK, Nrf2, HO-1, NQO1 and $\alpha$-tubulin were immunodetected using specific antibodies. (b) Relative NQO1 promoter activity in WT and Jdp2 KO MEFs in the presence of TPA at the indicated exposure times. After $24 \mathrm{~h}$ of culture, TPA (10 ${ }^{-6} \mathrm{M}$ ) was added, transfectants with pGL4-hQR25-luciferase were incubated for an additional $48 \mathrm{~h}$ and luciferase activity was measured $(n=3)$ as described in Materials and Methods. (c) Effect of Nrf2 on NQO1 promoter activity. pGL4-hQR25-luciferase ( $400 \mathrm{ng}$ ) plus $0-100 \mathrm{ng}$ of pcDNA3-Nrf2 were transfected into WT and Jdp2 KO MEFs $\left(5 \times 10^{4}\right)$. One day after transfection, cells were collected and luciferase activity was measured. Values from a representative experiment are given as mean \pm S.D. ( $n=3$ ). (d) Effect of JDP2 on ARE activity in the presence of Nrf2. Jdp2 KO MEFs $\left(5 \times 10^{4}\right)$ was transfected with $400 \mathrm{ng}$ of pGL4-hQR25-luciferase, $50 \mathrm{ng}$ of FLAG_S-Nrf2 and pcDNA-Jdp2, respectively, as indicated. One day after transfection, cells were collected and luciferase activity was measured $(n=3) .{ }^{* *} P<0.01$. (e) Protein detection of FLAG-Nrf2 and JDP2 in WT and Jdp2 KO MEFs using western blotting. The cellular lysates from WT and Jdp2 KO MEFs $(40 \mu \mathrm{g})$ were separated on SDS-PAGE and then transferred onto a membrane. FLAG, Jdp2 and $\beta$-actin were immunodetected using specific antibodies, which were described in Materials and Methods. Lane number corresponded to each lane in d. (f) Effect of siRNA specific for Nrf2 and Jdp2 on ARE activity. WT and Jdp2 KO MEFs ( $5 \times 10^{4}$ cells) were transfected with 30 pmol of siRNA specific for Nrf2 or Jdp2 and $200 \mathrm{ng}$ of pGL4-hQR25-luciferase plasmid as described in the text. After exposure for $30 \mathrm{~h}$, luciferase activity was measured $(n=3)$. The same amount of nonspecific double-stranded RNA was used as a negative control (NS). ${ }^{\star \star} P<0.01$. (g) Inhibition of the expression of Nrf2 and Jdp2 proteins by siRNA. WT and Jdp2 KO MEFs $\left(5 \times 10^{4}\right.$ ) were transfected with $30 \mathrm{pmol}$ of active siRNA and the control siRNA, together with reporter plasmids. After treatment for $30 \mathrm{~h}$, cell extracts were analyzed by immunoblotting using Nrf2, Jdp2, IgG or $\beta$-actin antibodies. Lane 1, without siRNA; lane 2, with siRNA; and lane 3, with NS siRNA 